\title{
Artificial Insemination and its Importance in Marine Crustaceans: A Review
}

\author{
Chandan Haldar*, Shyam Kumar and Raju Ram \\ ICAR Central Institute of Fisheries Education, India
}

*Corresponding author: Chandan Haldar, ICAR Central Institute of Fisheries Education, Mumbai, Maharashtra, 400061 India

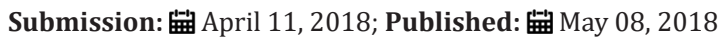

\begin{abstract}
Artificial Insemination (AI) is a tool for genetic manipulation in the crustacean stock including shrimp and crabs. It is seen as one of the means for propagating shrimp culture to new areas by controlled reproduction. But there are few studies on the reproduction and embryology of marine crustaceans whereas other groups of freshwater crustaceans have received much attention, and their embryology is better documented. So for the proper development of the marine production it is now necessary to focus on the artificial insemination technique in marine species also. In the current review we have discussed on the different tool and techniques used in artificial insemination of marine crustaceans. In future, more number of research and development works must be conducted to increase the production of marine crustaceans by controlled reproduction through artificial insemination.
\end{abstract}

Keywords: Artificial insemination; Electro-ejaculation; Marine crustaceans; Reproduction; spermatophore; (glue) a-cyanoacrylate

\section{Introduction}

In the recent years, with the advent of aquaculture of shrimp and other economically important crustacean species, interest in the methods of controlling reproduction in them has gained great impetus. The successful domestication of any species is facilitated by gaining control over its reproductive biology.

The artificial insemination (AI) is the deliberate introduction of sperm into a female genital for the purpose of completing a pregnancy through in vivo fertilization by means other than sexual intercourse. It is a fertility treatment practiced in a lot of animals for artificial breeding. Sandfier \& Smith (1979) have attempted to produce the culture suited strains through hybridisation in fresh water prawns by using the spermatophores of macrobrachium and Palaemonetes sp. by ejaculating by the electrical induction [1].

Chow et al. (1982) used $\alpha$-cyanoacrylate adhesive to attach the spermatophores to the female sternum as they do not spawn their eggs immediately after mating. So it is necessary that the sperms need to be transferred to them in a convenient and protective packet that can be stored until its needed. The mating occurs with the ventricle surface of the male and the female apposed .The spermatophore is extruded through the gonophore located at the base of the fifth walking leg by the contraction of striated muscle located around the distal vas deferens [2]. The transfer of spermatophore is added by the modified first pair of pleopods of the male which are rigid and contoured to hold the spermatophore. The female store spermatophore internally in a small pouch (thelycum or spermatheca) found in the midline on the ventral surface posterior to the gonopores. When the spermis present in the seminal receptacle, they are found along the wall farthest from the openings [3]. The spermatophore is held in this location by the large and brown plug that probably originates in segments of the vas deferens and hardens after transferring into to the female. The hard plug is very protective and is not essential for the sperm survival of the female that is artificially inseminated lack this plug, yet they may fertilize eggs $[4,5]$. The female does not release all the sperm from the seminal receptacle during a single spawning. The female artificially inseminated with banked spermatophore have successfully fertilized eggs, indicating that banked sperm retains their fertility. The sperm collected by electrical stimulation placed under paraffin oil and maintained at $4-7{ }^{\circ} \mathrm{C}$ contained morphologically normal sperm capable of undergoing normal acrosome reaction after 289 days of storage [6].

\section{Artificial Insemination by an Electrical Method}

A technique for electrically inducing spermatophore extrusion from live lobsters Homarus americanus (American lobster) is done by using a $12 \mathrm{~V}$ stimulus applied around the coxa of the fifth walking leg, a single spermatophore is extruded through the gonopore. The sperm in extruded spematophoresare morphologically normal and undergo normal acrosome reactions. The technique delivers a ready source of viable sperm for in vitro studies on fertilization and for artificial insemination of freshly molted female lobsters. The spermatophore itself is a natural package for sperm storage and may be useful in the future development of lobster sperm banks. A technique has been established for artificially inseminating newly 
molted female lobsters (Homarus). In Homarus, females normally mate within 24-48hr of a molt however; females have also been reported to mate while in a hard shell (intermolt) condition.

Three categories of females were artificially inseminated:
A. Laboratory-born H. Americanus
B. Wild-born Homarus americanus, and
C. Hybrids of $\mathrm{H}$. Americanus Q X H. gammarus and the reciprocal cross.

Artificial inseminations were achieved on females in almost all cases within the first $24 \mathrm{hr}$ but always within the first $48 \mathrm{hr}$ next molting. Directly prior to artificial insemination, one or two spermatophores were collected from a laboratory- maintained male (H. americunus) using an electrical extrusion technique. The spermatophore(s) was positioned on the tip of a bamboo stick until placed in the thelycum of the female. Inseminations were done by placing the female ventral side up on the surface of a laboratory bench [7].

The annulus, which is soft after a molt, was untied by implanting a small screwdriver parallel to the anterior-posterior axis and rotating it 900 . The spermatophore(s) was released into the thelycum through the opening, the screwdriver was removed so that the annulus closed, and the female was returned to her tank. Most females received one spermatophore, but occasionally two were introduced into the thelycum of females with a carapace length $>90 \mathrm{~mm}$. Artificially inseminated females were placed on a short-day photoperiod (8hr light/day) for 100-120 days and then transferred to long days (16hr of light/day). Spawning occurred 60-180 days following long-day onset. Egg samples were collected from the pleopods or from the tank bottom at various intervals after spawning. To determine if fertilization had occurred, either fresh or fixed eggs were examined with a dissecting microscope for evidence of normal cleavage and embryonic development. Eggs were subsequently sampled and monitored for the appearance of eyespots. Eggs were followed through larval development and monitored for hatching.

\section{Purpose of Artificial Insemination}

A. These techniques could be appreciated in experimental and commercial aquaculture; they ensure that a high-quality spermatophore will be relocated to the female and that the father of choice can be used.

B. In natural matings, some males will not mate with freshly molted females in captivity.

C. The artificial insemination procedure requires very little time. It can be completed in $10-15 \mathrm{~min}$ compared to $30 \mathrm{~min}$ to several hours required for natural mating.

D. The reproductive cycle of the female lobster is relatively long, it is especially important that a high-quality spermatophore be transported to the female of this species at each moult.
E. Studies proved that artificial insemination in penaeid shrimp shown to extrude fertilized eggs in a high percentage.

F. Decapods can easily be artificially inseminated with a high probability that fertilization will occur.

Artificial Insemination and Spawning of Pacific White Shrimp Litopenaeus Vannamei: Implications for A Selective Breeding Program

Maternal half-sib families by mating one female with two different males within a 2 -wk spawning period. The artificial insemination (AI) technique apply to produce these families relied on the deduction of both spermatophores from a single male and the application of the spermatophores over the thelycum of a ripe female. In an effort to make the best use of the number of half-sib families and reduce the time that families are produced, a different AI technique was used to produce paternal half-sib families. With this technique, each of the two spermatophores from a single male was manually extruded and placed on the thelycum of two different females.

The advantages of natural mates over artificial insemination are a greater number of naupli produced per spawn and decreased labour costs. However, the male is unknown and this may be important information for selective breeding programs. The specific males are mated with specific females by AI to produce half/ full-sib families for estimation of genetic parameters, including heritability estimates, phenotypic and genetic variation and phenotypic and genetic correlations.

\section{Double Spermatophore Technique: (Used to Produce Maternal Half-Sib Families)}

A. Capture a female with full ovarian development (in stage IV to V), visually identifiable by the well-developing ovaries which are thick from the posterior edge of the carapace through the posterior end of the abdomen.

B. Identify a male with fully developed spermatophores and manually eject both spermatophores by applying gentle pressure to the base of the outside corner of the spermatophore until it slips out of the genital pore. Healthy spermatophores show no signs of melanization, are white in colour, faintly swelled and are stiff to touch.

C. Carefully hold the ripe female so that her thelycum is wide-open. The fourth and fifth sets of pereopods should be engaged posteriorly and held against the ventral surface of her abdomen.

D. Place the first spermatophore anterior to the thelycum between the base of the third and fourth pereopods vertical to the long axis of the body. Return the fourth set of pereopods to their normal position, securing the first spermatophore in place.

E. Place the second spermatophore posterior to the thelycum between the base of the fourth and fifth pereopods 
perpendicular to the long axis of the body. Place the female in a spawning tank overnight. The insemination process should be finalised in less than $1 \mathrm{~min}$ to reduce stress to the female.

\section{Single Spermatophore Technique: (Used to Produce Paternal Half-Sib Families)}

The procedure for the single spermatophore technique is identical to that of the double spermatophore technique through step 3.

A. Place a single spermatophore between the thumb and index finger with firm constant pressure being applied from the bottom (closed end) toward the top (open end) of the spermatophore. This pressure ruptures the sperm sac and liberates a sperm mass that forms a droplet between the thumb and index finger

B. Hold the female securely in the position described in step 3 and carefully place the sperm mass inside the thelycum by inverting the forceps. The thelycum serves as the seminal receptacle and is enclosed by the coxae of the third and fourth set of pereopods and also partially by the ventral setae of these structures. After the sperm mass is correctly positioned, return the pereopods to their normal position.

\section{Conclusion}

Crustacean genetic engineering or genetic manipulation techniques are of a recent origin with most of the work being carried out on the applied aquaculture aspects, including bloodstock development, heritability, and hybridization. The primary techniques for stock improvement are stock selection, selective breeding hybridization and sex control. But, artificial insemination (AI), enhancements in the phenotype and genotype traits, disease resistance. growth rates, and a number of other characters. Difficulties such as disconnected nature of knowledge, and trouble in synchronization of mating behaviour of some commercially significant decapods can be positively tackled through AI - the key to successful breeding programme. So, AI can be useful in increasing production from a maturation laboratory and so assist in closing the life cycle of such species. But there are few number of research programmes reported on artificial insemination in crustacean. So more number of research and development works must be conducted in future to make the technique helpful in the domestication efforts for shrimp stocks and also in propagating them to new areas by controlled reproduction. From the foregoing account it is evident that although AI has great significance for inter- and intra-specific hybridization, the technique needs further refinement in order to match the success rates achieved by natural mating, spawning, and hatching.

\section{Reference}

1. Aiken DE, Waddy SL, Moreland K, Polar SM (1984) Electrically induced ejaculation and artificial insemination of the American lobster Homarusamericunus. J Crustacean Biology 4(4): 519-527.

2. Dall W, Hill BJ, Rothlisberg PC, Sharples DJ (1990) The biology of the Penaeidae. Advances in Marine Biology 27: 3-4.

3. Hetzel DJ, Crocos PJ, Davis GP, Moore SS, Preston NC, (2000) Response to selection and heritability for growth in the Kuruma prawn, Penaeus japonicus. Aquaculture 181(3-4): 215-223.

4. Pawar R, Mohiuddin MI (2010) Artificial insemination in penaeid shrimps. Journal of the Indian Fisheries Association 27: 19-25.

5. Sandifer PA, Smith TIJ (1979) A method for artificial insemination of Macrobrachium prawns and its potential use in inheritance and hybridization studies. Pro World Maricult Soc 10(1-4): 403-418.

6. Talbot P, Thaler C, Wilson P (1986) Artificial insemination of the American lobster (Homarus). Molecular Reproduction and Development 14(1): 25-31.

7. Yano I, Tsukimura B, Sweeney JN, Wyban JA (1988) Induced ovarian maturation of Penaeusvannamei by implantation of lobster ganglion. Journal of the World Aquaculture Society 19(4): 204-209.
Creative Commons Attribution 4.0 International License

For possible submissions Click Here

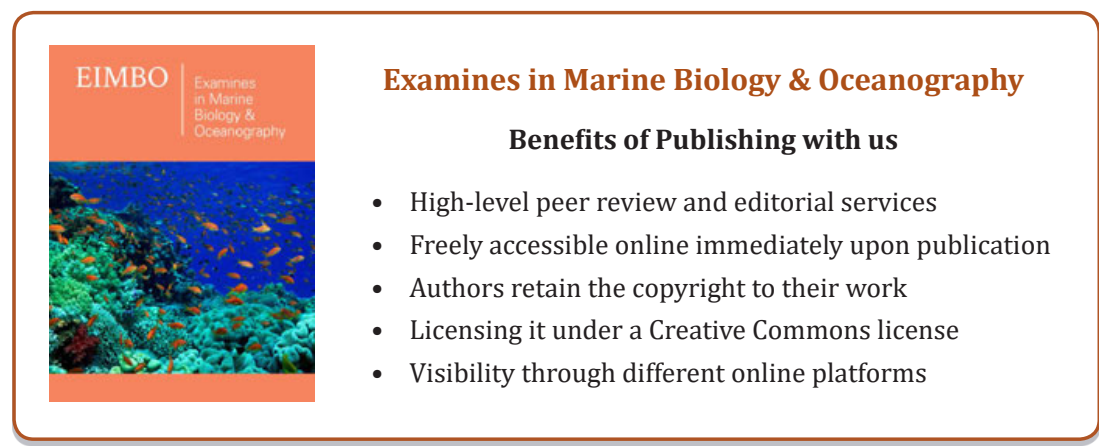

